\title{
El factor regulación como determinante del consumo energético y de las emisiones de $\mathrm{CO}_{2}$
}

\author{
M. Ángeles Fernández López ${ }^{a, *}$, Yolanda Fernández Fernández ${ }^{b}$, \\ David González Hernández ${ }^{\mathrm{a}}$ y Blanca Olmedillas Blanco ${ }^{\mathrm{b}}$
}

\author{
a Departamento de Economía y Empresa, Universidad Católica de Ávila, Ávila, España \\ b Departamento de Análisis Económico, Teoría Económica e Historia Económica, Universidad Autónoma de Madrid, Madrid, España
}

Recibido el 15 de julio de 2013; aceptado el 3 de diciembre de 2013

Disponible en Internet el 22 de enero de 2014

\section{CÓDIGOS JEL \\ Q40; \\ Q56}

\section{PALABRAS CLAVE}

Emisiones de $\mathrm{CO}_{2}$; Producto interior bruto;

Consumo de energía;

Planes nacionales

de asignación

\section{JEL CLASSIFICATION \\ Q40; \\ Q56}

\section{KEYWORDS}

$\mathrm{CO}_{2}$ emissions;

Gross domestic

product;

Energy consumption;

National allocation

plans

\begin{abstract}
Resumen En los últimos años la literatura económica ha prestado especial atención a la relación entre emisiones contaminantes, consumo de energía y actividad económica. Este trabajo pretende profundizar en dicha relación, prestando especial atención al posible efecto que la aplicación de los planes nacionales de asignación ha tenido sobre el comportamiento emisor y energético de los países analizados. En concreto, se estudia si han logrado ser efectivos a la hora de reducir 2 variables clave como son las emisiones de $\mathrm{CO}_{2}$ y el consumo de energía, incluso cuando se han producido caídas en la actividad económica debido a la crisis de los últimos años. Los países que se han tenido en cuenta para este trabajo son: España, Francia, Alemania, Italia, Portugal y Reino Unido. El periodo considerado es 2000-2010, y se utilizan datos publicados por la Unión Europea relativos a emisiones, asignaciones de derechos, consumo de energía y nivel de producción de los países analizados.

(c) 2013 Asociación Cuadernos de Economía. Publicado por Elsevier España, S.L. Todos los derechos reservados.
\end{abstract}

The regulation factor as a determinant of energy consumption and $\mathrm{CO}_{2}$ emissions

Abstract The economic literature has recently been paying special attention to the relationship between pollutant emissions, energy consumption, and economic activity. This work aims to look deeper into that relationship, in particular to the possible effect that National Allocation Plans (NAPs) have had on the pollutant and energy behaviour of the countries analysed, especially as regards reducing the key variables; $\mathrm{CO}_{2}$ emissions and energy consumption, even although the economic activity has declined due to the recent crisis. The countries studied for this work were: Spain, France, Germany, Italy, Portugal, and the United Kingdom. The study

\footnotetext{
* Autor para correspondencia.

Correos electrónicos: angeles.fernandez@ucavila.es, mangelesfl@gmail.com (M.Á. Fernández López).
} 
period was from 2000 to 2010, and all the data have been published by the European Union, as regards emissions, rights allowances, energy consumption, and production level of the countries analysed.

๔ 2013 Asociación Cuadernos de Economía. Published by Elsevier España, S.L. All rights reserved.

\section{Introducción}

En los años previos a la crisis económica, la mayoría de economías desarrolladas centraron su atención en la lucha contra el cambio climático, y dada la variedad de contaminantes ${ }^{1}$, la lucha se centró, fundamentalmente, en la reducción de los gases de efecto invernadero (GEI) por ser contaminantes con efectos globales, a largo plazo y de difícil reducción. En este contexto, el fomento de la eficiencia energética $-y$ por tanto de la eficiencia emisorase convirtió en una cuestión clave para conseguir la reducción de los GEI. Sin embargo, en los últimos años la caída en la actividad económica ha desplazado este objetivo a un segundo plano, y al mismo tiempo esa caída en la actividad ha ido acompañada de caídas en los niveles de emisiones contaminantes.

Son numerosos los estudios cuyo objetivo es esclarecer la relación entre crecimiento económico, emisiones contaminantes y consumo de energía, y todos ellos muestran la complejidad de las citadas relaciones. La mayoría de ellos tratan de explicar la relación entre emisiones contaminantes y el crecimiento económico teniendo en cuenta 3 factores: tecnológicos, estructurales y preferencias, utilizando distintas técnicas de descomposición y herramientas de análisis.

La creación en 2005 del mercado europeo de derechos de emisión supuso el primer compromiso cuantitativo preciso sobre limitación de las emisiones de GEI. Los derechos de emisión se asignaban a través de los Planes Nacionales de Asignación (PNA). Finalizado el segundo PNA (2008-2012), consideramos que para explicar la evolución de las emisiones y el consumo de energía en el marco de la Unión Europea (UE) habría que considerar un nuevo factor: el factor regulación que capte la eficacia de los PNA en la consecución de la reducción de las emisiones contaminantes y del consumo de energía.

Por tanto, el objetivo de este trabajo es valorar la eficacia de los PNA en la reducción de emisiones y el ahorro energético, teniendo siempre en cuenta la relación de ambas variables con el crecimiento económico. Nuestro análisis se centra en 6 países pertenecientes a la Unión Europea (España, Francia, Alemania, Italia, Portugal y Reino Unido) entre los años 2000 y 2010.

El trabajo se estructura en 4 apartados. En el primero se describe la relación entre el crecimiento económico y la intensidad emisora y energética. En el segundo apartado se analizan los resultados agregados de los PNA mediante el

\footnotetext{
${ }^{1}$ Algunos con efectos globales y otros con efectos más regionales y/o locales; algunos con muchos focos de emisión y otros con las emisiones más concentradas.
}

balance entre asignaciones y emisiones por países y sectores. En el tercero se utilizan 2 modelos econométricos para analizar la importancia del PIB y de los PNA en la evolución de las emisiones de $\mathrm{CO}_{2}$ y del consumo de energía. Por último, comentamos las principales conclusiones.

\section{Crecimiento económico e intensidad energética y emisora}

\subsection{Revisión de la literatura}

Las políticas de eficiencia energética se han convertido en una prioridad en todos los países debido a su doble papel: en la reducción de las emisiones contaminantes ${ }^{2}$ y en la reducción de la dependencia energética ${ }^{3}$. Según la Comisión Europea (2011a, 2011b), la eficiencia energética tendrá efectos positivos en el crecimiento económico y en la creación de empleo. También es una de las formas más rentables de reforzar la seguridad del abastecimiento energético, de reducir las emisiones de GEI y de otras sustancias contaminantes, así como de hacer el proceso energético más asequible para los consumidores.

Desde mediados de los años noventa, la mayor parte del debate sobre los efectos ambientales del crecimiento económico se ha centrado en la hipótesis de la «curva de Kuznets ambiental» (CKA). Esta hipótesis sostiene que la relación entre la renta per cápita y las emisiones contaminantes puede representarse por una $U$ invertida, de forma que en una primera fase las emisiones tendrían una relación creciente con la renta hasta alcanzar un nivel crítico de renta per cápita a partir del cual las emisiones contaminantes disminuirían a medida que se producen posteriores incrementos en la renta.

Sin embargo, los resultados empíricos acerca de la CKA no son concluyentes y ninguno de los contaminantes considerados en la literatura cumple de forma clara la hipótesis de la CKA (Ekins, 1997; Papayotou, 1997; De Bruyn y Heintz, 1999; Stern y Common, 2001; Díaz-Vázquez, 2009). Por tanto, la hipótesis no es generalizable, en particular para los contaminantes con efectos globales, a largo plazo y cuya reducción es más complicada (caso del $\mathrm{CO}_{2}$ ).

Se deduce, por tanto, que la relación entre crecimiento económico y emisiones contaminantes es compleja, por lo que su estudio requiere un análisis detallado de las relaciones entre los indicadores medioambientales y el PIB. Una cuestión importante, en lo que respecta a los indicadores,

\footnotetext{
2 Favoreciendo la lucha contra el cambio climático.

3 Sobre todo, de combustibles fósiles.
} 


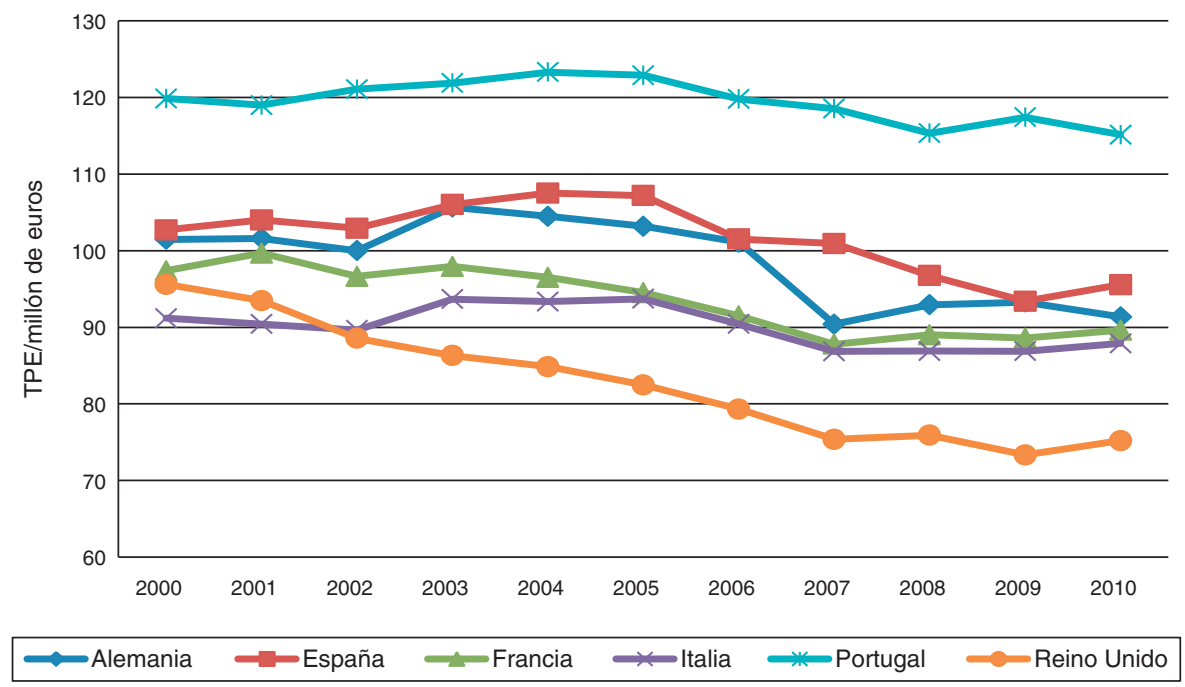

Fuente: Elaboración propia a partirde Eurostat (2013)

Figura 1 Intensidad energética.

Fuente: Elaboración propia a partir de Eurostat (2013).

es que hay que tener en cuenta que la mejora de un indicador podría, además de coexistir, explicarse por el comportamiento negativo de otro indicador; por ejemplo, las emisiones asociadas al uso de combustibles fósiles podrían reducirse por el creciente uso de energía nuclear.

Ante esta cuestión, algunos estudios consideran alguna proxy de presión ambiental total. Entre otras alternativas, Suri y Chapman (1998) utilizan el uso global de energía, Mielnik y Goldemberg (1999) utilizan el índice de carbonización (emisiones por unidad de energía consumida) y Ang (1999) utiliza la intensidad energética (consumo de energía por unidad de PIB).

Para explicar la relación entre emisiones contaminantes y crecimiento económico hay que tener en cuenta varios factores: tecnológicos, estructurales y preferencias. En la literatura se distinguen numerosos estudios cuyo objetivo es recoger estos factores utilizando diferentes técnicas de descomposición y herramientas de análisis (Grossman y Krueger, 1991; Roca y Padilla, 2003; Díaz-Vázquez y Cancelo, 2010).

\subsection{Un nuevo factor explicativo: factor regulación}

Centrándonos en las emisiones de GEI y dada su dimensión transfronteriza, su solución -y más concretamente su nivel de provisión- no se puede decidir a nivel individual, sino que requiere un acuerdo internacional voluntario en el que participen numerosos países. En este sentido, la creación en 2005 del mercado europeo de derechos de emisión supuso el primer compromiso cuantitativo preciso sobre limitación de las emisiones de GEI. Los derechos de emisión se asignan a través de los PNA. En los inicios del tercer PNA (20132020) consideramos que, para explicar la evolución de las emisiones en el marco de la UE, habría que considerar un factor regulación que capte la eficacia de los PNA en la consecución de la reducción de las emisiones contaminantes y del consumo de energía ${ }^{4}$.

En concreto, el interés de este trabajo se centra en considerar un factor adicional a los ya mencionados: el factor regulación. Se trata de observar si los PNA han propiciado el desacoplamiento entre emisiones y producción y entre consumo de energía y producción. Esto es, si los PNA han sido efectivos para reducir la intensidad emisora (emisiones por unidad de PIB) y la intensidad energética (consumo de energía por unidad de PIB). El estudio se centra en 6 países pertenecientes a la UE: España, Francia, Alemania, Italia, Portugal y Reino Unido, entre los años 2000 y 2010 . Todos los países analizados están sujetos a la misma normativa sobre el Comercio de derechos de emisión y han participado en las 2 convocatorias del mismo: la primera, que abarca los años 2005 a 2007, y la segunda, de 2008 a 2012.

Para poder analizar mejor la contribución de estos países al medio ambiente y su protección, es conveniente utilizar indicadores, no en términos absolutos, sino por unidad de PIB producida. De esta forma, la intensidad emisora se define como las toneladas de $\mathrm{CO}_{2}$ emitidas por unidad de PIB generado por una economía, y la intensidad energética, como las toneladas de petróleo equivalentes utilizadas para producir una unidad de PIB. En ambos casos, la unidad de PIB es un millón de euros en términos reales.

El análisis de ambos indicadores (figs. 1 y 2) permite observar que Portugal presenta la producción menos eficiente energéticamente y la más emisora.

Respecto al gasto energético, la economía que consume menos toneladas de petróleo equivalente para generar una unidad de PIB es la del Reino Unido. Pero respecto a las emisiones, es Francia la que emite menos GEI por unidad de producción en todo el período analizado.

\footnotetext{
${ }^{4}$ Para una revisión de las políticas de mitigación del cambio climático en la UE, ver Soria y Saveyn, 2010.
} 


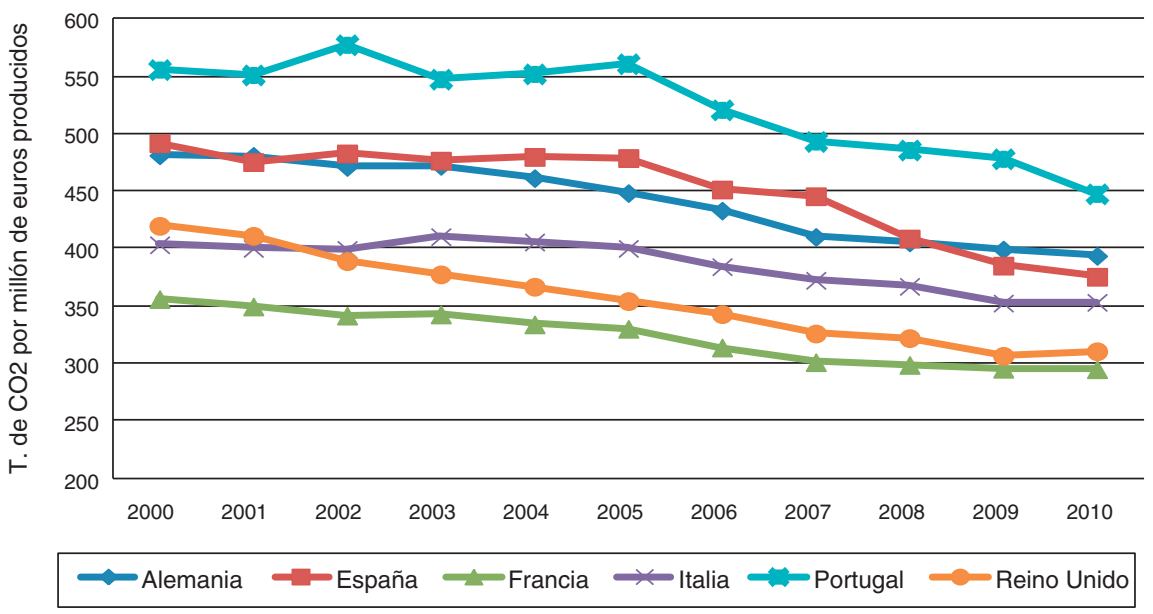

Fuente: Elaboración propia a partir de Comisión Europea y Eurostat (2013)

Figura 2 Intensidad emisora.

Fuente: Elaboración propia a partir de la Comisión Europea (2013) y Eurostat (2013).

En todos los países analizados llama poderosamente la atención la evolución de dichos indicadores, que disminuyen de manera significativa a partir de 2005, año de entrada en vigor del primer período del Comercio de derechos de emisión. Así lo muestran las tasas de variación interanuales medias entre 2000-2004 y 2005-2010 para todos los países excepto Reino Unido, cuya tasa de variación es incluso mayor en el periodo 2000-2004 (tabla 1).

Teniendo en cuenta que las tasas de variación interanuales medias del PIB real en los períodos 2000-2004 y 2005-2010 son positivas, la tabla 1 evidencia que existe un desacoplamiento entre las emisiones y la producción, por una parte, y entre el consumo de energía y la producción, por otra. Cabe señalar que, al analizar el desacoplamiento de una economía, debe de tenerse en cuenta la existencia del efecto denominado «efecto rebote» (Schipper, 2000), que nos indica que el propio aumento de la eficiencia en el uso de un recurso natural podría estimular su demanda, reduciendo o incluso anulando en casos extremos el efecto reductor del aumento de eficiencia. Por ejemplo, si aumenta la eficiencia de los vehículos y consumen menos gasolina, se abaratan los desplazamientos, lo que puede generar un aumento en la demanda de los mismos, compensando en parte el ahorro inicial.
Dada la evolución de la intensidad emisora y energética para el periodo considerado procede, a continuación, analizar brevemente el resultado agregado de los PNA por países y sectores.

\section{Resultados agregados de los Planes Nacionales de Asignación}

La idea que subyace a la creación del mercado de derechos de emisión (regulado por la Directiva 2003/87/CE de la Unión Europea [2003]) es que, como en cualquier mercado, si se produce escasez de oferta, el precio subirá. $Y$ en este caso concreto de derechos, se busca una subida suficientemente alta como para incentivar que los agentes participantes encuentren más beneficioso cambiar de sistema productivo a uno energéticamente más eficiente y menos contaminante. La forma en que dicho mercado ha sido puesto en práctica ha comenzado por la aplicación, en cada país participante, de unos límites a la contaminación recogidos en los PNA.

En relación con el límite de las emisiones de $\mathrm{CO}_{2}$ recogido en los planes, denominadas emisiones verificadas, hay que señalar que en ninguno de los países analizados llega al

Tabla 1 Tasas de variación interanuales medias (TVIM)

\begin{tabular}{llllll}
\hline & \multicolumn{2}{c}{ TVIM 2000-2004 } & & \multicolumn{2}{c}{ TVIM 2005-2010 } \\
\cline { 2 - 3 } \cline { 5 - 6 } & Intensidad energética & Intensidad emisora & & Intensidad energética & Intensidad emisora \\
\hline Alemania & 0,73 & $-1,05$ & $-2,41$ & $-2,57$ \\
España & 1,15 & $-0,61$ & $-2,27$ & $-4,75$ \\
Francia & $-0,22$ & $-1,59$ & $-1,06$ & $-2,23$ \\
Italia & 0,59 & 0,15 & $-1,28$ & $-2,47$ \\
Portugal & 0,71 & $-0,17$ & $-1,30$ & $-4,41$ \\
Reino Unido & $-2,94$ & $-3,32$ & $-1,83$ & $-2,60$ \\
\hline
\end{tabular}

Fuente: Elaboración propia a partir de los datos de Eurostat (2013). 
$50 \%$ de las emisiones totales del país. Los sectores afectados son básicamente generación-combustión (de más de $20 \mathrm{MW}$ ) e industria, quedando fuera del comercio el resto de emisiones de los sectores llamados difusos (muchos de ellos de gran importancia en el total de emisiones contaminantes: sector residencial, agricultura, transporte...).

En todos los países analizados las asignaciones se han realizado en función de las emisiones históricas ${ }^{5}$, dejando una reserva para nuevos entrantes en cada uno de los periodos de comercio. Ese dato de emisiones históricas ha hecho que el mayor volumen de asignaciones recaiga en el sector de generación-combustión. La excepción es Francia, donde la fuente principal de energía es nuclear, mucho menos emisora de $\mathrm{CO}_{2}$.

Respecto a la evolución de las asignaciones, en el periodo de prueba del comercio (2005-2007) hubo una sobreasignación de derechos. A ello se le unió la imposibilidad de trasladar derechos de este periodo al siguiente, lo que facilitó la caída del precio hasta casi cero al final del año 2007 (Ciscar y Soria, 2009: 47-48). Esto dificultó alcanzar los objetivos finales, pues el precio del derecho era tan bajo que parece poco probable que se cambie de sistema energético y productivo 6 .

En un intento de corregir la situación anterior, en el segundo periodo de comercio (2008-2012) se produjo una fuerte reducción en el volumen de derechos asignados, y esta caída se aplicó fundamentalmente al sector de generación-combustión. Posiblemente la mayor exigencia de reducción de emisiones a este sector esté basada en 2 motivos: por un lado, la búsqueda de un cambio de sistema energético (a otro menos basado en el consumo de combustibles fósiles) y, por otro, la elevada concentración de emisiones en pocos agentes contaminantes, lo que facilita el control.

En el tercer periodo de comercio (que acaba de iniciarse en 2013), además de incluir nuevos sectores sometidos a verificación, los derechos han dejado de asignarse gratuitamente (como sucedía en los 2 planes anteriores). Se espera que con estas mejoras el comercio dé resultados positivos y sirva de señal para generar cambios de comportamiento de los agentes contaminantes.

En la figura 3 se presenta el balance entre asignaciones y emisiones por países y sectores. El signo de la cobertura absoluta, entendida como la diferencia entre asignaciones y emisiones verificadas, permite conocer la posición inicial de cada país/sector en el mercado de derechos de emisión.

- La existencia de un equilibrio entre ambos supondría un resultado de cero.

- Si el resultado es positivo, el país/sector en cuestión disfruta de un exceso de derechos de emisión. El superávit de derechos supondría la posibilidad de venderlos en el mercado de derechos de emisión, con la intención de obtener ingresos adicionales a la actividad productiva propia de cada instalación. En el primer periodo, entre 2005 y 2007, era la única opción. A partir de 2008, los derechos

\footnotetext{
${ }^{5}$ Ver Riestra et al. (2009: 64-65) y Neuhoff et al. (2006).

${ }^{6}$ Para un estudio detallado del primer periodo del comercio de derechos de emisión, ver Ellerman y Joskow, 2008.
}

excedentarios pueden venderse o acumularse para periodos posteriores.

- Si el resultado es negativo, muestra la falta de derechos asignados para cubrir las emisiones. Si hay déficit, las instalaciones de cada país pueden acudir al mercado de derechos de emisión como demandantes de los mismos ${ }^{7}$.

A nivel teórico, cabría esperar una situación deficitaria generalizada, para que a los países/sectores les suponga un coste internalizar la externalidad medioambiental. Si se trata de un valor positivo, el mecanismo puesto en marcha puede suponer ingresos extra para el país/sector en cuestión.

Queda patente que la emisión de $\mathrm{CO}_{2}$ a la atmósfera solo supone un coste evidente para el sector de generacióncombustión en Alemania, Reino Unido, España e Italia. Sin embargo, está suponiendo una fuente posible de ingresos para el sector industrial en general y para el sector de generación-combustión francés. Es decir, la asignación de derechos de emisión en estos casos no ha generado escasez, sino que ha sido lo suficientemente cuantiosa como para cubrir la externalidad y generar ingresos por la venta de los derechos excedentarios, lo que equivale a una «subvención encubierta», eso sí, a precio de mercado.

No debemos olvidar también el efecto de la crisis económica, que se observa sobre todo en 2009, con menores emisiones de $\mathrm{CO}_{2}$ a la atmósfera por la ralentización en la producción. Esto ha permitido que algunas instalaciones obtengan ingresos en el mercado de derechos de emisión, utilizándolos como fuente de financiación de su actividad principal.

\section{Estimaciones del efecto de los Planes Nacionales de Asignación sobre las variables estudiadas}

\subsection{Datos}

Para la estimación se han utilizado datos de panel. Las variables utilizadas para la estimación (PIB y emisiones de $\mathrm{CO}_{2}$ ) proceden de Eurostat (2013). El PIB real, expresado en millones de euros de 2005, ha sido calculado a partir de los índices de volumen encadenados ofrecidos en Eurostat. Las emisiones de $\mathrm{CO}_{2}$ están expresadas en miles de toneladas de $\mathrm{CO}_{2}$ equivalentes. El consumo de energía, expresado en millones de toneladas de petróleo equivalentes, se ha obtenido de $B P$ Statistical Review of World Energy (BP, 2012).

Como ya se indicó, la muestra de países seleccionada incluye 6 países de la UE: Alemania, España, Francia, Italia, Reino Unido y Portugal. El período muestral es de 2000 a $2010^{8}$.

\footnotetext{
7 También pueden realizar acciones que les proporcionen derechos adicionales de emisión, denominados CER y ERU. Si no cumplen su compromiso (o límite), se prevé la imposición de una multa a la instalación.

${ }^{8} \mathrm{La}$ falta de datos de emisiones de $\mathrm{CO}_{2}$ posteriores a 2010 ha limitado el análisis a ese año.
} 


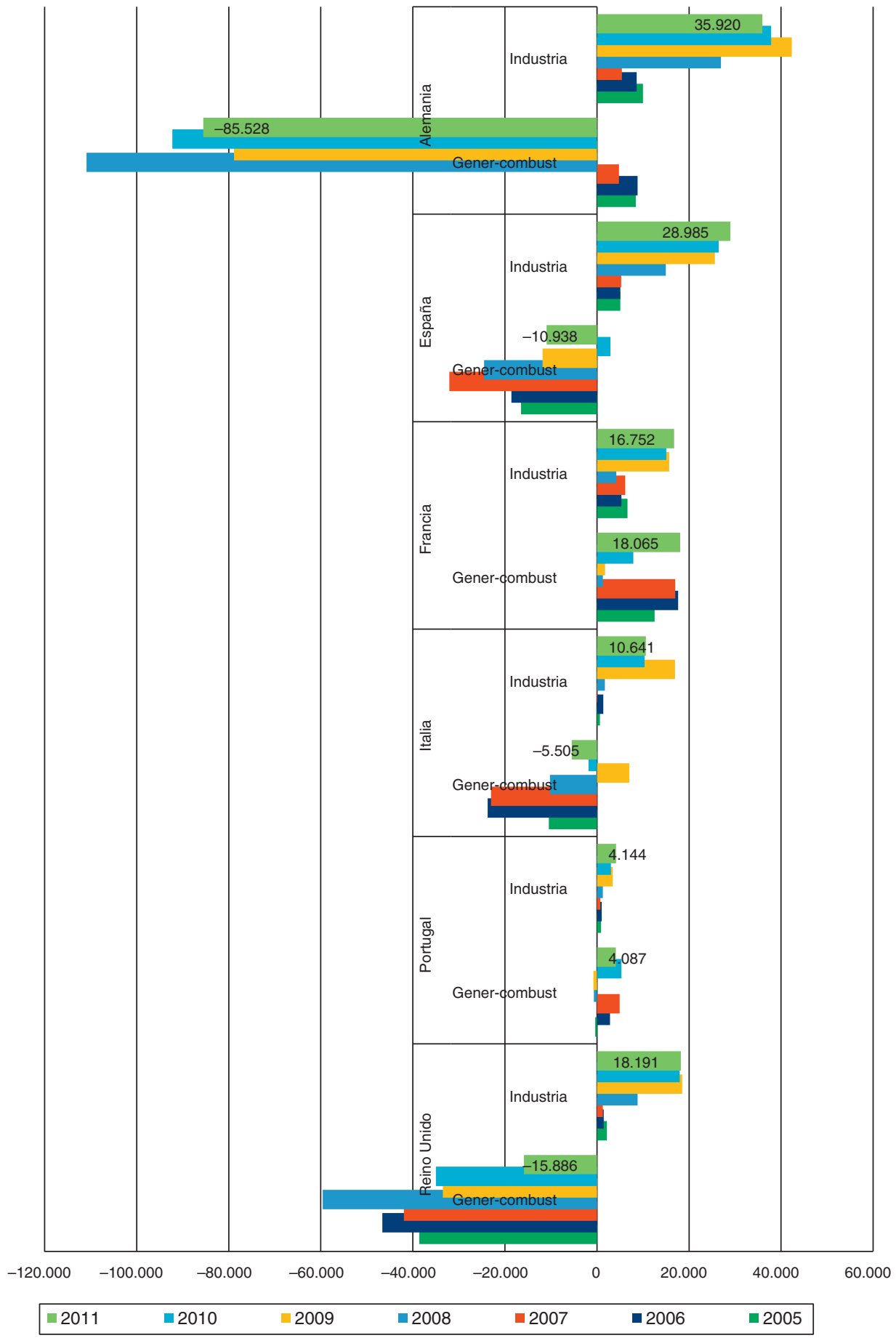

Fuente: Elaboración propia a partir de datos de la Comisión Europea (2013).

Figura 3 Balance entre asignaciones y emisiones (en toneladas).

Fuente: Elaboración propia a partir de datos de la Comisión Europea (2013).

\subsection{Modelos econométricos}

Para analizar si los PNA han tenido influencia en la evolución de las emisiones y del consumo de energía final, se han realizado 2 estimaciones econométricas que se presentan a continuación.
La relación empírica en la que se basa este estudio consiste en establecer la relación tanto de las emisiones totales como del consumo de energía con el PIB real. Esta relación se completa con una constante, $\gamma$, que recoge otros efectos exógenos no incluidos bajo la producción, y el error, e. 


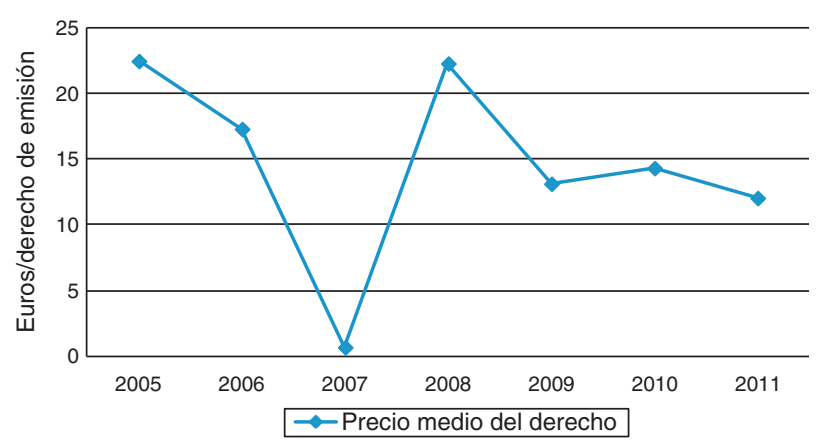

Fuente: Elaboración propia a partir de los datos de Bluenext (2013)

Figura 4 Precio diario del derecho de emisión (media anual). Fuente: Elaboración propia a partir de los datos de Bluenext (2013).

Se han tomado las series en logaritmos, lo que permite interpretar los coeficientes en términos de elasticidades; el coeficiente de cada una de las variables independientes indica aproximadamente el porcentaje en que se modificaría la variable dependiente con un $1 \%$ de desviación en la variable independiente.

La relación de las variables «emisiones»y «consumo de energía» con la renta depende, fundamentalmente, de 3 factores: preferencias, parámetros tecnológicos y regulaciones (Esteve y Tamarit, 2012). Nuestro interés se centra en analizar cómo determinados cambios en la política económico-ambiental han influido en estas relaciones. En concreto, la importancia de la entrada en vigor de los PNA, la primera fase para el período 2005-2007 y la segunda fase para 2008-2012.

Sería razonable considerar el precio del derecho como variable explicativa en ambas regresiones. Sin embargo, y como muestra la figura 4, el precio diario del derecho (promedio anual) cae hasta aproximarse a cero en 2007, cuando finaliza el primer período de aplicación de los PNA. Las causas principales son 2: el exceso de derechos asignados y la imposibilidad de transmitir los derechos sobrantes al segundo periodo (2008-2012). A partir de 2008, cuando se produce una nueva asignación, el precio del mismo vuelve a incrementarse. La desaceleración económica ha dado lugar a una menor necesidad de derechos, lo que ha presionado el precio a cotas inferiores a los 15 euros por tonelada emitida.

Las intensas variaciones experimentadas por el precio del derecho de emisión en un periodo de tiempo relativamente breve como es 2005-2010 hacen que, para este análisis, se haya optado por incluir, en lugar de los precios del derecho, los PNA como variables que influyen en la relación entre PIB y emisiones de $\mathrm{CO}_{2}$ por una parte (modelo 1 ) y PIB y consumo de energía por otra (modelo 2 ). La consideración de los PNA se realiza a través de 2 variables cualitativas o dicotómicas.

El modelo 1 a estimar es el siguiente:

$\ln E T_{i t}=\alpha_{i}+\beta_{1} \cdot \ln P I B_{i t}+\beta_{2} \cdot P N A_{1}+\beta_{3} \cdot P N A_{2}+u_{i t}$

Donde:

$E T$ : emisiones totales de $\mathrm{CO}_{2}$ (miles de toneladas); $\alpha$ : intersección para cada individuo; PIB: PIB real (millones de euros); $P N A_{1}$ : variable dicotómica que toma el valor 1 para el periodo de prueba o primer periodo del Comercio de derechos de emisión (2005-2007); $P N A_{2}$ : variable dicotómica que toma el valor 1 para los años disponibles del segundo periodo del Comercio de derechos de emisión (2008-2012); $\beta_{1}, \beta_{2}, \beta_{3}$ : coeficientes a estimar; $u_{i t}$ : término error; $i$ : país (Alemania, España, Francia, Italia, Portugal y Reino Unido); $t$ : año (periodo de estudio 2000-2010).

Para estimar la función anterior se ha optado en un primer momento por un modelo de efectos fijos ${ }^{9}$. En la tabla 2 se muestran los resultados obtenidos. El coeficiente positivo de 0,417 para el PIB nos indica que la evolución de las emisiones totales está ligada a la actividad económica. El coeficiente de $-0,035$ para la variable $\mathrm{PNA}_{1}$ muestra que el periodo 2005-2007 ha tenido un efecto negativo en la evolución de las emisiones. El coeficiente de $-0,127$ para la variable $\mathrm{PNA}_{2}$ indica que el segundo periodo del Comercio de derechos de emisión ha tenido un papel más importante en la reducción de las emisiones. Por tanto, podemos deducir que, aunque gran parte del comportamiento de las emisiones totales viene explicado por la actividad económica, la implantación del Comercio de derechos de emisión ha contribuido a reducir las emisiones totales de $\mathrm{CO}_{2}$.

El contraste $F$ de significación de los efectos fijos, $F(5$, $57)=155,51$, confirma la presencia de estos efectos e indica que es preferible el modelo de efectos fijos a la regresión agrupada (pooled data). Los efectos individuales pueden hacer referencia a la distinta forma en la que se ha implantado el Comercio de derechos de emisión en cada país. Cada Estado miembro ha disfrutado de cierta autonomía a la hora de diseñar su PNA.

También se ha estimado un modelo de efectos aleatorios. No obstante, aplicando el test de Hausman ${ }^{10}$ se puede rechazar la hipótesis nula de que los efectos individuales no están correlacionados con los regresores. Por tanto, es recomendable utilizar el modelo de efectos fijos.

La validez del modelo de efectos fijos está limitada por los problemas de autocorrelación ${ }^{11}$, heterocedasticidad ${ }^{12} \mathrm{y}$ correlación contemporánea ${ }^{13}$.

Por último, se ha estimado un modelo por mínimos cuadrados generalizados factibles (feasible generalizad least squares, FGLS) en el que se corrigen los problemas señalados. En este caso, el coeficiente asociado al PIB es mucho mayor que en el caso del modelo de efectos fijos. Las variables $\mathrm{PNA}_{1}$ y $\mathrm{PNA}_{2}$ siguen siendo válidas ${ }^{14}$ y los coeficientes presentan valores similares al modelo de efectos fijos.

En resumen, los resultados de la estimación muestran una relación positiva entre el PIB real y las emisiones de $\mathrm{CO}_{2}$. El coeficiente $\beta_{1}$ indica que la elasticidad entre las 2 variables es positiva y cercana a la unidad, de forma que las emisiones de $\mathrm{CO}_{2}$ habrían aumentado a medida que aumenta el

\footnotetext{
${ }^{9}$ Estimador intragrupos que proporciona el programa estadístico Stata.

10 Valor del estadístico chi-cuadrado $(3)=11,05$.

11 Test de Wooldridge para autocorrelación en datos de panel. $\mathrm{F}(1$, 5) $=34,445$.

12 Prueba modificada de Wald. Valor del estadístico chi-cuadrado (6) $=12,59$.

13 Prueba de Breusch-Pagan. Valor del estadístico chi-cuadrado $(15)=38,435$.

$14 \mathrm{PNA}_{1}$ sigue siendo válida, a un nivel de significancia al $10 \%$.
} 
Tabla 2 Resultados de las estimaciones del modelo 1

\begin{tabular}{lccc}
\hline Variables & Efectos fijos & Efectos aleatorios & $\begin{array}{c}\text { Mínimos cuadrados } \\
\text { generalizados factibles } \\
\text { (FGLS) }\end{array}$ \\
\hline PIB $($ In) & & $0,785(13,49)$ & $0,872(131,51)$ \\
PNA $_{1}$ & $0,417(3,34)$ & $-0,064(-5,69)$ & $-0,035(-1,74)$ \\
PNA $_{2}$ & $-0,035(-2,58)$ & $-0,157(-13,89)$ & $-0,101(-4,13)$ \\
Constante & $-0,127(-9,02)$ & $2,144(0,81)$ & $0,910(8,95)$ \\
Test F de significación conjunta o Wald & $7,22(4.18)$ & 274,54 g. de $\mathrm{l} .=3$ & $17.297,81 \mathrm{~g}$. de $\mathrm{l} .=3$ \\
\hline
\end{tabular}

Los datos entre paréntesis corresponden al estadístico t de Student.

Fuente: Elaboración propia.

PIB. Por tanto, no habría evidencia de desvinculación entre emisiones y renta.

Por el contrario, influyen con signo negativo los PNA cuyos coeficientes $\beta_{2}$ para el período 2005-2007 y $\beta_{3}$ para el período 2008-2012 nos indican que la entrada en vigor de los mismos ha favorecido la disminución de las emisiones, especialmente en el segundo PNA, en el que el mercado estaba más establecido y se habrían superado algunas limitaciones ocurridas en la primera fase, como la sobreasignación de derechos que provocó un exceso de oferta de los mismos con la consecuente caída del precio del derecho y, en definitiva, del coste de emisión. Por tanto, el Comercio de derechos de emisión, y sobre todo la aplicación de los PNA como forma de internalizar la externalidad ambiental, ha tenido efectos positivos. En este sentido pueden esperarse resultados más eficientes, siempre que se genere una mayor escasez de derechos.

Modelo 2. Para analizar la eficacia de los PNA como instrumento para lograr una economía menos intensiva en consumo de energía primaria se estima la siguiente función expresada en términos logarítmicos:

$\ln C E_{i t}=\delta_{i}+\gamma_{1} \cdot \ln P I B_{i t}+\gamma_{2} \cdot P N A_{1}+\gamma_{3} \cdot P N A_{2}+\mu_{i t}$

Donde:

$C E$ : consumo de energía primaria; $\delta$ : intersección para cada individuo; PIB: PIB real (millones de euros); $P N A_{1}$ : variable dicotómica que toma el valor 1 para el periodo de prueba o primer periodo del Comercio de derechos de emisión (2005-2007); $P N A_{2}$ : variable dicotómica que toma el valor 1 para los años disponibles del segundo periodo del Comercio de derechos de emisión (2008-2012); $\gamma_{1}, \gamma_{2}, \gamma_{3}$ : coeficientes a estimar; $\mu_{i t}$ : término error; $i$ : país (Alemania, España, Francia, Italia, Portugal y Reino Unido); $t$ : año (periodo de estudio 2000-2010).

Para estimar la función anterior se ha optado, al igual que antes, por un modelo de efectos fijos ${ }^{15}$. En la tabla 3 se muestran los resultados obtenidos. El coeficiente positivo de 80,586 para el PIB nos indica que la evolución del consumo de energía primaria está muy ligada a la actividad económica. El coeficiente de $-3,315$ para la variable $\mathrm{PNA}_{1}$ muestra que el periodo 2005-2007 ha tenido un impacto negativo en el consumo de energía. El coeficiente de $-12,574$ para la

\footnotetext{
15 Estimador intragrupos que proporciona el programa estadístico Stata.
}

variable $\mathrm{PNA}_{2}$ indica que el segundo periodo del Comercio de derechos de emisión ha tenido un papel más importante en la reducción del mismo. Este análisis sugiere que, al igual que en el modelo anterior, la implantación del Comercio de derechos de emisión ha contribuido a reducir el consumo de energía primaria.

El contraste $F$ de significación de los efectos fijos, $F(5$, $57)=542,80$, confirma la presencia de estos efectos e indica que es preferible el modelo de efectos fijos a la regresión agrupada (pooled data). Los efectos individuales pueden hacer referencia al mix energético de cada país.

También se ha estimado un modelo de efectos aleatorios. Utilizando la prueba del multiplicador de Lagrange para efectos aleatorios desarrollada por Breush y Pagan, podemos rechazar la hipótesis nula; la varianza del error es igual a 0 , por lo que existen diferencias entre el modelo estimado por mínimos cuadrados ordinarios.

Aplicando el test de Hausman ${ }^{16}$ no se puede rechazar la hipótesis nula de que los efectos individuales no están correlacionados con los regresores. Por tanto, se ha optado por utilizar el modelo de efectos aleatorios.

La validez del modelo de efectos fijos está limitada por los problemas de heterocedasticidad ${ }^{17}$ y correlación contemporánea $^{18}$.

Como en el caso anterior, se ha estimado también un modelo por FGLS en el que se corrigen los problemas señalados $^{19}$. En este caso, el coeficiente asociado al PIB es mayor que en el caso del modelo de efectos fijos y aleatorios. Las variables $\mathrm{PNA}_{1}$ y $\mathrm{PNA}_{2}$ siguen siendo válidas y los coeficientes presentan valores similares a los modelos anteriores.

Los resultados obtenidos indican que, aunque la evolución del consumo de energía está muy ligada a la actividad económica, el Comercio de derechos de emisión o, quizás más concretamente, la aplicación de los PNA ha contribuido a la reducción de este consumo energético, dado el signo negativo de ambos coeficientes. Como en el caso anterior, es mayor el efecto del segundo plan que el del primero.

\footnotetext{
16 Valor del estadístico chi-cuadrado (3) = 11,05.

17 Prueba modificada de Wald. Valor del estadístico chi-cuadrado (6) $=34,06$.

18 Prueba de Breusch-Pagan. Valor del estadístico chi-cuadrado (15) $=46,592$.

19 También se ha estimado un modelo con errores estándar corregidos para panel con resultados similares.
} 
Tabla 3 Resultados de las estimaciones del modelo 2

\begin{tabular}{lccc}
\hline Variables & Efectos fijos & Efectos aleatorios & $\begin{array}{l}\text { Mínimos cuadrados } \\
\text { generalizados factibles } \\
\text { (FGLS) }\end{array}$ \\
\hline PIB $($ ln) & & $89,534(6,02)$ & $96.738(207,12)$ \\
PNA $_{1}$ & $80,586(3,62)$ & $-4,01(-1,96)$ & $-3,499(-4,40)$ \\
PNA $_{2}$ & $-3,315(-1,37)$ & $-13,313(-6,38)$ & $-13,296(-16,65)$ \\
Constante & $-12,574(-5,02)$ & $-1.043,802(-5,06)$ & $-1.144,004(-5,814)$ \\
Test F de significación conjunta o Wald & $-920,105(-2,99)$ & 59,45 g. de l. $=3$ & $43.370,99 \mathrm{~g}$. de $\mathrm{l} .=3$ \\
\hline
\end{tabular}

Los datos entre paréntesis corresponden al estadístico t de Student o test de significación individual.

Fuente: Elaboración propia.

No obstante, en futuros estudios sería conveniente ampliar el número de países para corregir alguno de los problemas detectados en la estimación.

En resumen, y teniendo en cuenta los 2 modelos especificados, los resultados obtenidos indican que tanto las emisiones como el consumo de energía primaria están ligados a la actividad económica. Sin embargo, los PNA han contribuido, por una parte, a reducir las emisiones totales, y por otra, a introducir cambios en el sistema productivo, de manera que aumentar la producción requiera ahora menores incrementos en el consumo de energía.

\section{Conclusiones}

La reducción de $\mathrm{GEI}$ y en particular del $\mathrm{CO}_{2}$ se ha convertido en un objetivo clave para las economías desarrolladas. Con la puesta en marcha en 2005 del Comercio de derechos de emisión, la UE estableció el firme compromiso de combatir el cambio climático.

En los inicios del tercer PNA (2013-2020), el objetivo de este trabajo es aportar evidencia empírica de la influencia del que hemos denominado factor regulación, considerado a través de los PNA, en la evolución de las emisiones de $\mathrm{CO}_{2}$ y del consumo de energía, teniendo en cuenta la relación de ambas variables con el crecimiento económico.

Del análisis realizado para el período 2000-2010 de 6 países europeos (Alemania, España, Francia, Italia, Portugal y Reino Unido) destacan las conclusiones siguientes:

- En primer lugar, el análisis de la evolución de la intensidad emisora y energética para los países considerados muestra la existencia de un cierto desacoplamiento entre emisiones y producción y entre consumo de energía y producción, siendo más intenso este desacoplamiento en el período 2005-2010, período en el que el Comercio de derechos de emisión está en funcionamiento.

- En segundo lugar, y en lo que a los resultados agregados de los PNA se refiere, el balance entre asignaciones de derechos y emisiones verificadas por países y sectores muestra que todas las reducciones de asignaciones de derechos de emisión han recaído en el sector generación-combustión frente al industrial, lo que corrobora la importancia de las políticas de eficiencia energética en la reducción de emisiones.

- En tercer lugar, y tras la estimación econométrica de 2 modelos para contrastar la influencia de los PNA y del
PIB en la evolución de las emisiones (modelo 1) y consumo energético (modelo 2 ), hay que destacar que en ambos modelos se evidencia que tanto la evolución de las emisiones como la del consumo de energía están ligadas a la actividad económica, como muestran las estimaciones de los coeficientes $\beta_{1}$ y $\gamma_{1}$ al tomar valores positivos.

- En ambos modelos también se observa que la aplicación de los PNA ha favorecido la reducción tanto de las emisiones como del consumo de energía, como constatan los coeficientes estimados $\beta_{2}, \gamma_{2}$ para el primer $\mathrm{PNA}_{1}$ y $\beta_{3}, \gamma_{3}$ para el segundo $\mathrm{PNA}_{2}$, que toman valores, todos ellos, negativos. Además, los coeficientes para el segundo $\mathrm{PNA}_{2}$ son de mayor cuantía que los del primer $\mathrm{PNA}_{1}$. Estos resultados muestran que el mercado de derechos de emisión estaba más afianzado, superando algunas limitaciones importantes ocurridas en la primera fase.

- También es destacable, con respecto a los coeficientes que representan la influencia de los PNA, que son mayores en el caso del modelo 2 (estimación de la influencia de los PNA en el consumo energético). Esto puede ser consecuencia de que la mayor reducción de asignaciones (básicamente en el segundo periodo) ha incidido en el sector generación-combustión.

De estos resultados se deduce la eficacia del Comercio de derechos de emisión en la reducción de las emisiones de $\mathrm{CO}_{2}$ y del consumo de energía, por lo que cabría esperar que el tercer PNA (2013-2020), en el que se han introducido cambios significativos conducentes a un mejor funcionamiento, dé lugar a mejores resultados. Por otro lado, cabría esperar también que esta internalización del coste externo de las emisiones de $\mathrm{CO}_{2}$ incentive actuaciones (adopción de tecnologías menos contaminantes, mayor I+D...) que conduzcan a un cambio en el modelo productivo menos contaminante y de mayor eficiencia energética.

\section{Financiación}

Los autores agradecen la financiación recibida de la Junta de Castilla y León a través del proyecto UCA191A11-1.

\section{Bibliografía}

Ang, B.W., 1999. Is the energy intensity a less useful indicator tan the carbon factor in the study of climate change? Energy Policy 27, 943-946. 
Bluenext [consultado Ene 2013]. Disponible en: http://energyprices.enerdata.net/markets/carbon-prices.html

BP, Statistical Review of World Energy. 2012 [consultado Ene 2013]. Disponible en: http://www.bp.com/statisticalreview

Ciscar, J.C., Soria, A., 2009. La política climática europea. Papeles de Economía Española 121, 45-53.

Comisión Europea, 2011a. Propuesta de Directiva del Parlamento Europeo y del Consejo, relativa a la eficiencia energética y por la que se derogan las Directivas 2004/8/CE y 2006/32/CE. COM, 370.

Comisión Europea, 2011b. Plan de Eficiencia Energética. Comunicación de la Comisión al Parlamento Europeo, al Consejo, al Comité Económico y Social Europeo y al Comité de las Regiones. COM, 109.

Comisión Europea, 2013. Community Transaction Log [consultado Ene 2013]. Disponible en: http://ec.europa.eu/ environment/ets/

De Bruyn, S.M., Heintz, R.J., 1999. The environmental Kuznets curve hypothesis. En: Van den Bergh, J. (Ed.), Handbook of Environmental and Resource Economics. Edward Edgar, Cheltenham, pp. 656-677.

Díaz-Vázquez, M.R., 2009. The dissociation between emissions and economic growth: The role of shocks exogenous to the environmental Kuznets curve model. Applied Econometrics and International Development 9, 31-42.

Díaz-Vázquez, M.R., Cancelo, M.T., 2010. Análisis de los factores determinantes de la evolución de las emisiones de $\mathrm{CO}_{2}$ y de azufre en países de la OCDE mediante una descomposición econométrica. Revista de Economía Mundial 26, 85-106.

Ekins, P., 1997. The Kuznets curve for the environment and economic growth: Examining the evidence. Environment and Planning A 29, 805-830.

Ellerman, A., Joskow, P., 2008. The European Union's Emissions Trading System in Perspective. Pew Center on Global Climate Change, Washington, D.C.

Esteve, V., Tamarit, C., 2012. Threshold cointegration and nonlinear adjustment between $\mathrm{CO}_{2}$ and income: The Environmental Kuznets Curve in Spain 1857-2007. Energy Economics 34, 2148-2156.
Eurostat, 2013 [consultado Ene 2013]. Disponible en: http://epp. eurostat.ec.europa.eu/portal/page/portal/statistics/search _database

Grossman, G., Krueger, A., 1991. Environmental Impacts of a North American Free Trade Agreement, NBER Working Paper n. 3914, noviembre. National Bureau of Economic Research, Cambridge, MA.

Mielnik, O., Goldemberg, J., 1999. The evolution of the carbonization index in developing countries. Energy Policy 27, 307-308.

Neuhoff, K., Ahman, M., Betz, R., Cludius, J., Ferrario, F., Holmgren, K., et al., 2006. Implications of announced phasell national allocation plans for the EU ETS. Climate Policy 6, 411-422.

Papayotou, T., 1997. Demystifying the Environmental Kuznets Curve: Turning a black box into a policy tool. Environment and Development Economics 2, 465-484.

Riestra, C., López, A., González, E., 2009. El $\mathrm{CO}_{2}$ y la industria en España: de la primera asignación de derechos a la etapa postKioto. Economía Industrial 371, 59-77.

Roca, J., Padilla, E., 2003. Emisiones atmosféricas y crecimiento económico en España. La curva de Kuznets ambiental y el protocolo de Kyoto. Economía Industrial 351, 73-86.

Schipper, L., 2000. On the rebound: The interaction of energy efficiency, energy use and economic activity. An introduction. Energy Policy 28, 351-353.

Soria, A., Saveyn, B., 2010. Present and Future of Applied Climate Mitigation Policies: The European Union, 207-217. En: Cerdá, E., Labandiera, X. (Eds.), Climate Change Policies. Global Challenges and Future Perspectives. Edwards Elgar, Cheltenham, UK.

Stern, D.I., Common, M.S., 2001. Is there an Environmental Kuznets Curve for sulfur? Journal of Environmental Economics and Management 41, 162-168.

Suri, V., Chapman, D., 1998. Economic growth, trade and the environment: Implications for the environmental Kuznets curve. Ecological Economics 25, 195-208. 